\title{
Strengthening vital statistics in Brazil: investigation of ill-defined causes of death and implications on mortality statistics
}

Elisabeth B Franca, Fatima Marinho de Souza, Lenice H Ishitani, Renato Teixeira, Celia L Szwarcwald

\begin{abstract}
Background The number of ill-defined causes of death (IDCD) has been a significant problem among registered causes of death in Brazil. The IDCD proportion was $14.3 \%$ in 2000 , with huge regional differences. In 2005 , the Brazilian government implemented a project in order to decrease the IDCD in states and municipalities in the poorest regions. This study aims to compare the distribution of causes of death between IDCD investigated and observed data.
\end{abstract}

Methods For each death certificate with IDCD all attempts were made by health-service professionals to trace existing information about the final disease and cause of death. The sources of information were hospital records, municipality health departments, autopsies, family health teams, and civil registry records. For deaths that occurred at home, verbal autopsy questionnaires were applied. The underlying cause of death was assigned using the documented evidence available.

Findings During 2006-10, 27.6\% (132056 of 478674) of IDCD were investigated, and 64.2\% (84751 of 132056) of these deaths were reclassified into a defined group of causes. Deaths from diabetes, neuropsychiatric, and maternal conditions occurred with a higher frequency among ill-defined causes investigated and were under-diagnosed in observed data. Injuries were also under-reported, responsible for $7.7 \%$ ( 6551 of 84751 ) of the diagnoses among IDCD in that period. Cancer and respiratory diseases occurred with a higher frequency among registered deaths than among IDCD $(16.3 \%$ vs $\mathbf{1 1 . 1} \%$ for cancer and 11.0 vs $7.8 \%$ for respiratory diseases) when compared with circulatory or endocrine diseases $(30 \cdot 6 \%$ vs $43 \cdot 1 \%$ for circulatory and $6 \cdot 2 \%$ vs $10 \cdot 2 \%$ for endocrine diseases).

Interpretation These results show that the distribution of the cause of deaths after investigation of IDCD was different from those in observed data. Therefore, these differences must be taken into consideration when making redistribution of ill-defined causes based on observed data to avoid bias. The investigation of IDCD is critical to the creation of a reference for applying corrections to the observed data as well as to strategically improve the quality of mortality data.

Funding Ministry of Health of Brazil.

Contributors

EBF and FMdS participated in the study design, data analysis and interpretation, and drafted the paper. RT, LHI, and CLS participated in the study design and performed data analysis and interpretation. All authors read, contributed to, and approved the final manuscript.

Conflicts of interest

We declare that we have no conflicts of interest.
Published Online June 17, 2013

\section{School of Medicine} (Prof E B Franca PhD), Research Group in Epidemiology and Health Evaluation, Federal University of Minas Gerais, Belo Horizonte, Brazil (LH Ishitani PhD, R Teixeira MPH); Pan American Health Organization, Washington, DC, USA (F Marinho de Souza PhD); and Oswaldo Cruz Foundation, Rio de Janeiro, Brazil (Prof C L Szwarcwald PhD)

Correspondence to: Elisabeth B Franca, School of Medicine, Federal University of Minas Gerais, Av. Alfredo Balena, 190/731, Belo Horizonte, Minas Gerais, Brazil efranca@medicina.ufmg.br 